\title{
Maler, Mörder, Mathematiker
}

Dieser Artikel skizziert eine Geschichte der Bilder, die wir uns vom Inneren des menschlichen Körpers machen. Er beleuchtet jeweils den emotionalen Kontext und die zugrunde liegenden Menschenbilder. Hauptpersonen sind, dem Wandel der Zeiten und dem Stabreim folgend: Maler, Mörder (korrekter: Hingerichtete, das heißt auch Diebe aus Not, uneheliche Frauen etc.), Mathematiker und Informatiker (Letztere subsumiert unter Mathematiker).

Im christlichen Kulturkreis war, bis in die Renaissance hinein, der Blick ins Innere des menschlichen Körpers mit Risiken behaftet. Die Vorschrift »Du sollst dir kein Bildnis machen« galt zwar nur für die Darstellung Gottes; aber die Kirche hatte schon früh das Verbot ausgeweitet auf Engel und andere Wesen des täglichen Umgangs. Um auf der sicheren Seite zu sein, begann man mit allegorischen Darstellungen des Inneren verstorbener Heiliger, etwa im Kontext der Vanitas Mundi.

Im islamischen Kulturraum, zumindest im größten Teil davon, galt sogar das Verbot jedweder Abbildung des Äußeren von »Lebewesen, die Gott erschaffen hat « (Koran) - was der arabischen Kunst lange Zeit nur die reichen Wunderwelten der geometrischen und floralen Muster offen hielt, die wir noch heute auf Kacheln, Teppichen oder Gobelins bestaunen können.

Seit der Renaissance widersetzten sich zunächst Maler, die zugleich anatomische Interessen hatten, dem ausdrücklichen Verbot der fast allmächtigen Kirche, schlichen sich nachts auf den Richtplatz und sezierten die Leichen der Gehenkten, natürlich ohne deren Einverständnis. Leonardo da Vinci (1452-1519), der wohl berühmteste Vorreiter dieser Entwicklung, schrieb in sein Tagebuch: »Und wenn dich solche Dinge interessieren, so hindert dich vielleicht Übelkeit, und wenn diese dich nicht hindert, so hindert dich vielleicht Angst, nachts mit solchen geschundenen und geteilten und schrecklich anzusehenden Toten zu sein; und wenn dich dieses nicht hindert, vielleicht fehlt dir dann die Meisterschaft der Zeichnung ...«(Zöllner, 400)

Garant der Wahrheit einer Darstellung war also die Meisterschaft der Zeichnung (Abbildung 1).

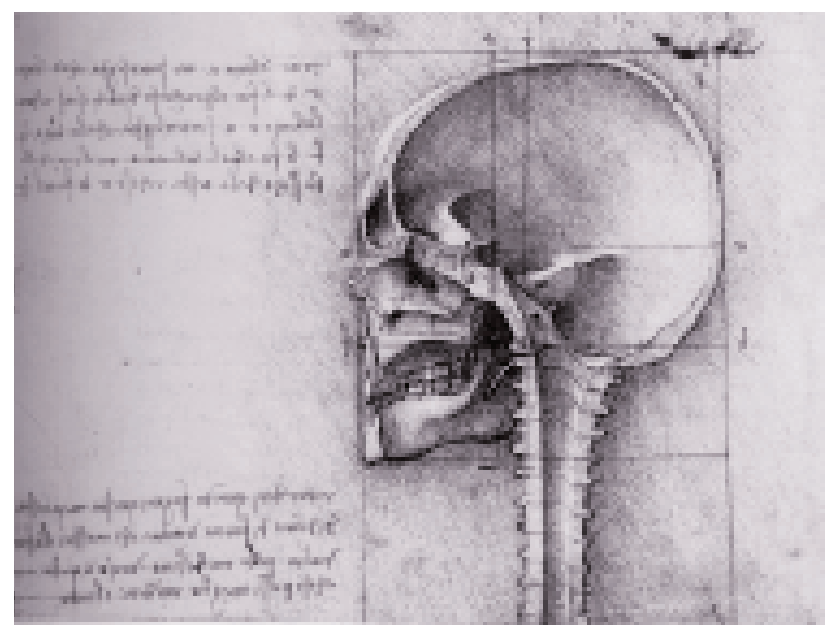

Abbildung 1: Leonardo da Vinci (1489): anatomische Studie eines Schädels. Vergleiche Abbildung 4

Auch als Sektionen schließlich ihren anerkannten Platz im akademischen Raum gefunden hatten, wurden vorzugsweise die Leichen Verurteilter seziert. In der holländischen Malerei hat sich das Anatomiegemälde sogar als eigenes Genre entwickelt.

Das Innere des Körpers 1656: der Mörder Joris Fonteijn Die meines Erachtens künstlerisch dichteste Darstellung der Situation ist Rembrandts Anatomie des Dr. Deyman aus dem Jahre 1656 (wohl zu unterscheiden von der glatteren Anatomie des Dr. Tulp aus dem Jahr 1632). Sektionen waren damals noch von einem Tabu umgeben; es war gegen jede Scham, die Leichname sehrbarer Bürger quasi in aller Öffentlichkeit in Teile zu zerstückeln. Keine Ehre hatten dagegen die zum Tode Verurteilten, ihre Leich- 
name wurden der Gilde der Chirurgen überantwortet (die damals noch nicht von den Pathologen oder Anatomen separiert waren). Ohne ordentliche Kühlungsmethoden mussten Sektionen natürlich sofort nach der Hinrichtung erfolgen.

Das in den Rembrandt-typischen Brauntönen gehaltene Ölgemälde (Abbildung 2) zeigt die Leiche des vielfachen Mörders Joris Fonteijn, auch Black Jack genannt, eines flämischen Schneiders, der am 17. Januar 1656 am Galgen starb. Das Bild ist im gleichen Jahr entstanden, infolge eines späteren Brandes jedoch stark beschädigt. Der Unterleib des Leichnams ist abgetrennt, die Eingeweide sind entfernt. Sein Gesicht, ins Zentrum des (unbeschädigten ursprünglichen) Gemäldes komponiert, starrt den Betrachter direkt an, klar kenntlich, entwürdigt. Der Anatom Deyman (halb verdeckt) hält die Dura Mater in seinen Händen, das Gehirn quillt aus dem geöffneten Schädel.

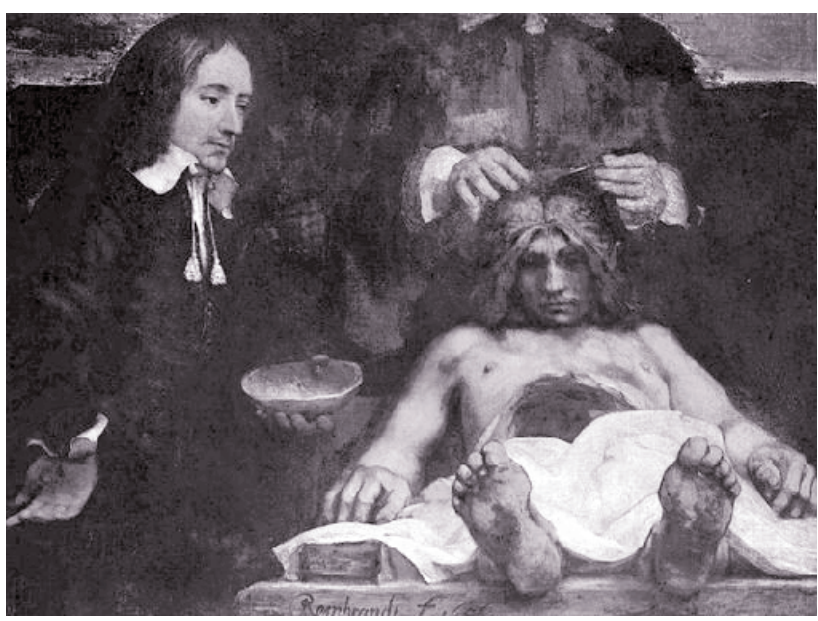

Abbildung 2: Rembrandt (1656): Die Anatomie des Dr. Deyman

Der mitfühlende Blick des damals 50-jährigen Rembrandt galt ausdrücklich dem Menschen allgemein, seiner Vergänglichkeit, seiner Zerstörbarkeit.

In unserer Zeit sezieren Generationen von Studierenden der Medizin an Leichen ... die immerhin zu Lebzeiten ihre Einwilligung gegeben hatten. Unsere Einstellung zu Sektionen wird kaum mehr öffentlich diskutiert oder gar reglementiert; der medizinische Nutzen überwiegt im öffentlichen Verständnis alle etwaigen Bedenken. Dennoch müssen auch heute noch fast alle Studierenden mit Gefühlen fertig werden, wie sie oben im historischen
Kontext beschrieben wurden - das bekannte Initiationsritual.

Die Entdeckung der Röntgenstrahlen am Ende des 19. Jahrhunderts revolutionierte die Welt der Bilder: Erstmals konnte das Innere des lebenden menschlichen Körpers direkt studiert werden (natürlich innerhalb gewisser Dosisgrenzen). Röntgenbilder waren allerdings nur Schattenbilder, was unbefriedigend war.

\section{Auftritt der Mathematik}

In dieser Situation trat der österreichische Mathematiker Radon (1887-1956) auf. Er untersuchte, rein abstrakt, die Frage: Lässt sich aus gemessenen Intensitäten vieler unterschiedlicher Strahlen, die ein Material durchdrungen haben, auf dessen Dichteverteilung im Innern schließen?

An Röntgenstrahlen hat er dabei gar nicht gedacht. Zur Lösung seines abstrakten Problems führte er eine heute nach ihm benannte Transformation ein. Im Jahre 1917 gelang ihm die Herleitung einer Umkehrformel, welche die obige Frage exakt beantworten sollte. Sein Beweis der Formel war so elegant, dass er auch heute noch zum Kanon gehört (siehe Natterer). Leider ist die klassische Radon-Formel für die Anwendung unbrauchbar: Schon kleine Fehler in den gemessenen Daten können das gesuchte Resultatbild vollständig verwischen. In der Sprache der Mathematik: Das Problem erwies sich als >schlechtgestellt $<$, was allerdings korrigierbar ist. Seitdem haben zahlreiche Mathematiker dieses Forschungsgebiet vorangetrieben, das im Deutschen irreführenderweise Computertomografie, im Englischen korrekter Computerized Tomography genannt wird, abgekürzt CT. Mit Blick auf die praktische Anwendung, etwa in der Medizin, spitzte sich das Problem rasch auf die Suche nach effizienten Algorithmen (das heißt Berechnungsmethoden) zu. Ein kurzer Exkurs für den interessierten Laien sei erlaubt: Gehen wir von einem Bild mit N x N Pixeln (i.e. digitalen Bildpunkten) aus, so ist ein Gleichungssystem mit $\mathrm{N}^{2}$ Unbekannten zu lösen, im technisch relevanten Fall $\left(\mathrm{N}=10^{24}\right)$ also mit zirka einer Million Unbekannten. Übliche Verfahren, die auf Gauß zurückgehen, würden $\sim \mathrm{N}^{6} \approx 10^{18}$ Rechenoperationen benötigen; sie nutzen die Spezialstruktur des Problems nicht aus und sind damit zu langsam.

Ohne Kenntnis der Radon'schen Arbeiten schlug der englische Ingenieur Hounsfield 1968 einen Algorithmus mit nur $\sim 50 \mathrm{~N}^{4}$ Operationen vor, ein echter Durchbruch für die damalige Zeit, und baute 1973 das erste kommer- 


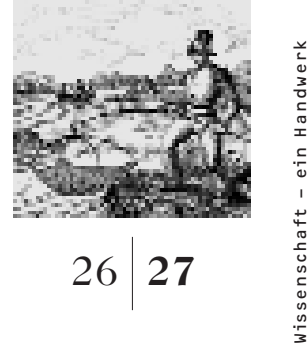

zielle CT-Gerät. Zusammen mit dem Physiker Cormack, der schon 1963/64 bahnbrechende Beiträge zum Thema CT geleistet hatte, erhielt er 1979 den Nobelpreis für Medizin.

In Kenntnis der Arbeiten von Radon publizierten 1974 die Mathematiker Shepp und Logan einen Algorithmus, der nur $\sim \mathrm{N}^{3} \approx 10^{9}$ Operationen benötigt (natürlich ohne den Nobelpreis zu bekommen). Ihre Methode findet sich heute noch in unseren CT-Geräten. Zur Berechnung von einer Million Unbekannten im typischen CT braucht ein marktüblicher PC nicht mehr als fünf Sekunden; die Methode von Hounsfield würde auf dem gleichen PC 70 Stunden geschätzte Rechenzeit >verbraten und wäre so für den klinischen Einsatz uninteressant.
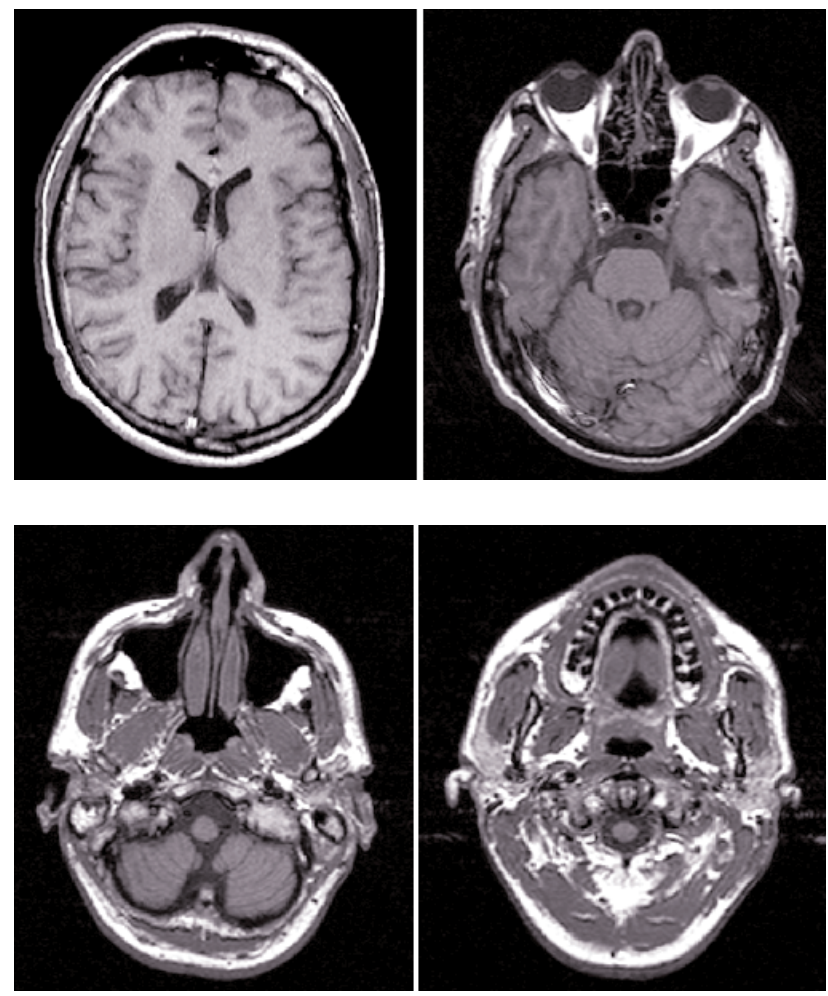

Abbildung 3: MRT-Bilder: planare Schnitte durch einen Kopf

Probleme dieses Typs treten inzwischen neben der CT auch in der Magnetresonanztomografie (MRT), der Positron-Elektron-Tomografie (PET) oder im Ultraschall auf; in allen diesen Fällen kann man auf die schnellen CT-Algorithmen oder ihre heutigen, noch effizienteren Nachfolger zurückgreifen. Inzwischen hat die medizinische Bildverarbeitung ihren festen Platz im klinischen Alltag (Abbildung 3). Als Grenze für den neugierigen
Blick ins Innere verbleiben noch die physiologisch maximal zumutbare Strahlenbelastung (bei CT und PET) sowie die begrenzte Auflösung.

Um swahre Bilder aus dem Innern des Menschen zu gewinnen, muss heutzutage niemand mehr sterben. Die begriffliche Nähe zur >minimalinvasiven Medizin` ist unübersehbar. Dennoch haben Hingerichtete längst nicht ausgedient, wie das folgende Beispiel zeigt.

\section{Das Innere des Körpers 1993:}

der Mörder Joseph Jernigan

Am 5. August 1993 wurde Joseph Paul Jernigan in Huntsville, Texas, mit der Giftspritze hingerichtet. Er hatte zwölf Jahre zuvor während eines Einbruchs einen alten Mann getötet. Jernigan vermachte seinen Körper testamentarisch dem Anatomical Board of the State of Texas. Der Mann wurde weltbekannt als der erste Visible Male. Der Anatom Vic Spitzer, einer der beiden Principal Investigators des Visible-Human-Projektes, äußerte sich zur Wahl von Jernigans Leichnam, wie von Wadman wörtlich zitiert: »It's very difficult to find an intact, nontraumatized, non-pathologic cadaver. I'm not condoning execution. But I don't believe in wasting resources either.»

In der Tat erfüllte der Leichnam nicht alle Kriterien: Er war zu übergewichtig (der Delinquent hatte im Gefängnis so stark zugenommen, dass er fast nicht in den Scanner passte); ihm fehlten ein Teil der Hoden, der Blinddarm und ein Zahn. Er war offenbar dennoch der beste verfügbare Kandidat innerhalb des Visible-HumanProjektes.

Das Anatomical Board übernahm den noch warmen Körper unmittelbar nach der Hinrichtung. Man brachte ihn zunächst in ein örtliches Bestattungsunternehmen, wo sein Körper in blaue Gelatine eingelegt wurde. Sodann wurde er in ein Charterflugzeug verfrachtet, nach Denver geflogen und dort im Colorado Medical Sciences Center abgeliefert. Etwa acht Stunden nach der Hinrichtung wurden die ersten medizinischen Bilder der Leiche aufgenommen: MRT-Scans und CT-Scans des gesamten Körpers; um diese Techniken erfolgreich anwenden zu können, durfte der Körper nicht zu lange tot sein. Anschließend wurde er tiefgefroren und in zirka 1800 Scheiben von $1 \mathrm{~mm}$ Dicke zersägt. Alle Schichten wurden einzeln fotografiert und digitalisiert, sämtliche Pixels sorgfältig elektronisch gespeichert.

Die Sache hatte eine lange Diskussion der ethischen Grundlagen zur Folge. Natürlich war Jernigan zum Zeit- 
punkt seines Vermächtnisses nicht klar, dass sein Körper digitalisiert werden würde, und schon gar nicht, dass seine Daten (seit November 1994) im Internet veröffentlicht würden, sogar mit Namensnennung. Allerdings hatte er im Gespräch mit dem Gefängnisgeistlichen und seinem Anwalt zweifelsfrei geäußert, er wolle »etwas zurückgeben für das, was er weggenommen hatte«.

Noch im gleichen Monat wurde eine 59-jährige anonyme Hausfrau aus Maryland zur ersten Visible Female. Sie war an Herz-Kreislauf-Versagen natürlich gestorben und hatte ihren Körper ebenfalls der Anatomie vermacht, diesmal in Kenntnis des Visible-Human-Projektes.

Heute sind die Daten des Visible Male (15 GByte) und der Visible Female (40 GByte) im Internet kostenfrei verfügbar: Nach Beantragung einer Lizenz von der US National Library of Medicine und unter Beachtung der Auflage einer korrekten Zitierweise können sie einfach heruntergeladen werden. Beide Visible-Human-Daten zusammen wurden inzwischen von Bremer Mathematikern um Heinz-Otto Peitgen mit Wavelet-Techniken derart komprimiert, dass sie auf zwei CD-ROMs erworben werden können. Inzwischen haben sich weitere Visible-Human-Initiativen wie eine Welle über den Globus ausgebreitet: unter anderem in Korea, China, Japan und Deutschland. Weitere Wellen von Massendaten rollen auf uns zu.

\section{Der virtuelle Mensch I: die Form}

Methoden wie CT oder MRT liefern nur zweidimensionale Bilder, bestenfalls Stapel davon, wie in Abbildung 3 gezeigt; beim Visible Human fällt zwar mehr Information an, aber ebenfalls zweidimensional. Die meisten Menschen sind jedoch dreidimensionale Wesen, wichtige Ausnahmen wurden von Marcuse beschrieben.

Erst in den letzten Jahren haben Informatik und Mathematik ausreichend schnelle und verlässliche Methoden bereitgestellt, um korrekte geometrische 3D-Modelle aus 2D-Bildstapeln zu generieren. So wurde der virtuelle Mensch geboren, der heute mit 3D-Brille in unseren gewohnten Sehkategorien zu betrachten und mit Space Mouse zu durchwandern ist.

Abbildung 4 zeigt einen sagittalen Schnitt durch die dreidimensionale Darstellung des Schädels eines Patienten in zweidimensionaler Projektion. Dieses Bild ist nun wahr im folgenden Sinn: Es beruht direkt auf Messdaten zu einem Individuum und wurde mit verlässlichen ma- thematischen Methoden berechnet, die aber Fehler nicht mit mathematischer Sicherheit ausschließen. Ein vergleichender Blick auf die Zeichnung von Leonardo da Vinci in Abbildung 1 lässt dennoch 500 Jahre erstaunlich zusammenschrumpfen.

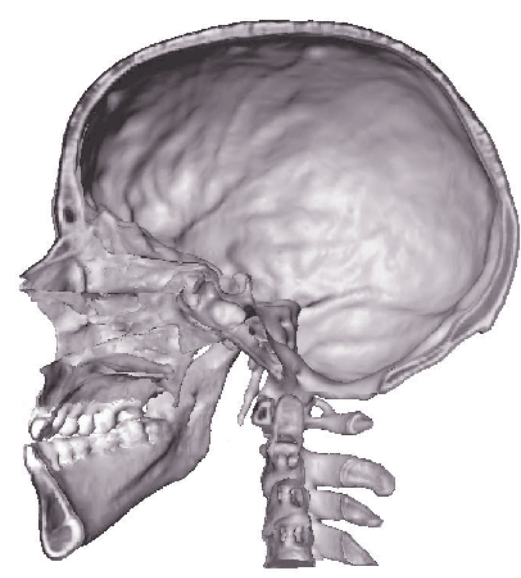

Abbildung 4: Virtueller Patient: Schnitt durch dreidimensionalen Schädel (Vergleiche Abbildung 1)

Die dreidimensionale Darstellung im Rechner gestattet, wenn geeignet programmiert, ein bequemes Herausgreifen einzelner grafischer Objekte oder, in anderen Worten, ein beliebiges Zerstückeln des Körpers, ohne auf Leichen zurückgreifen zu müssen! Der dazugehörige emotionale Schock wird von den damit arbeitenden Wissenschaftlern in der Regel nicht mehr wahrgenommen (die übliche Déformation professionelle), sehr wohl aber von Betrachtern, die mit solchen Techniken zum ersten Mal konfrontiert werden (meist durch ein Lachen ausgedrückt). Klar ist: Die Ausbildung von Studierenden der Medizin wird in Zukunft mehr und mehr auf virtuelle anatomische Atlanten in 3D zurückgreifen ${ }^{1}$.

Vorläufiger Höhepunkt dieser Entwicklung ist, innerhalb der Berlin-Brandenburgischen Akademie der Wissenschaften gewiss unbestritten, das >virtuelle Akademiemitglied : Abbildung 5 zeigt einen (vielleicht nicht sehr typischen) Ausschnitt, der aus einem heroischen Selbstversuch eines realen Akademiemitglieds stammt. Es sei ausdrücklich darauf hingewiesen, dass es sich hier um einen Mathematiker, aber (soweit bekannt) nicht um einen Mörder handelt; ansonsten sind die Daten natürlich anonym ${ }^{2}$. 


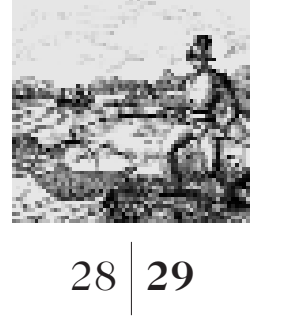

Mit der bequem im Rechner manipulierbaren visuellen Darstellung des Menschen sieht es nun so aus, als könnte Goethes Mephisto weiter spotten: "So hast du die Teile in deiner Hand, fehlt leider nur das geistige Band.»

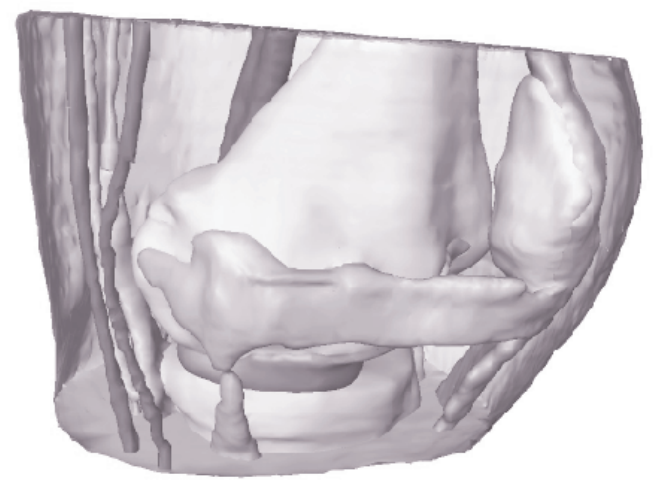

Abbildung 5: Virtuelles Akademiemitgliedsknie: dreidimensionale Darstellung des Kniegelenks mit Muskeln, Bändern und Blutgefäßen

\section{Der virtuelle Mensch II: die Funktion}

Goethes Spott ist jedoch nicht mehr so ganz berechtigt. Physiker, Ingenieure und Mathematiker gehen nämlich längst weiter: Sie versuchen, im Computer detailgenau die Funktion der einzelnen Teile des menschlichen Körpers zu modellieren und zu simulieren. Das ist schwer: Allein die Funktion der Niere ist so wenig verstanden, dass auch heute noch keine brauchbaren mathematischen Modelle existieren. Für die Medizintechnik, insbesondere für Therapie- und Operationsplanung, haben sich dennoch schon jetzt völlig neue Perspektiven eröffnet; Beispiele aus der Arbeitsgruppe des Autors sind etwa die Krebstherapie Hyperthermie oder die Mund-KieferGesichtschirurgie.

Am Ende dieses Wegs steht der Traum von einem modular aufgebauten Funktionsmodell des ganzen individuellen Menschen, das auch die systemische Rückwirkung auf die Teilmodelle berücksichtigt; in der Tat wäre erst mit dieser Kombination von Form und Funktion die Bezeichnung svirtueller Mensch gerechtfertigt. Trotz der fast erdrückenden Komplexität dieser interdisziplinären Aufgabe hat der Autor schon um 1985 für ein Projekt namens Homunculus geworben, das jedoch damals nicht genügend Anklang in der akademischen Community fand. Inzwischen jedoch ist die Zeit reif. So hat etwa der Bioingenieur Hunter, Auckland, mit einer großen Arbeitsgruppe begonnen, ein derartiges Projekt unter dem Namen Pysiome zu realisieren. In diesem Typ von Projekten wird der mathematischen Modellierung und effizienten Simulation eine Schlüsselrolle zukommen.

\section{Blicke hinter die Bilder}

Wie in fast allen Bereichen der modernen Natur- und Ingenieurwissenschaft kann auch in dem geschilderten unser Verständnis, gemeint als Ratio und Emotio, nicht mit dem Tempo der Entwicklung der Technologie Schritt halten. Deshalb sei hier kurz kontemplativ innegehalten.

Welches Menschenbild liegt hinter diesen Bildern vom Inneren des Menschen? Welche Perspektiven kommen auf uns zu? Ist der Mensch etwa wirklich auf dem Weg, sich nur noch als Summe seiner Teile zu begreifen? Haben wir nicht schon mehr als die >Teile in unserer Hand?

Um ins Innere des Körpers schauen zu können, benutzen wir Methoden der zerstörungsfreien Materialprüfung. Dies weckt das Bild vom Körper als $>$ Materialsammlung . Auch die objektorientierte 3D-Darstellung assoziiert den Menschen als Summe seiner Teile. Allerdings sind schon zahlreiche Wissenschaftler über dieses allzu simple Bild hinweg: Sie beschäftigen sich bereits mit der Modellierung der Funktion der Teile und ihres Wechselspiels. Der virtuelle Mensch, wie beschrieben, ist offenbar eine Konkretisierung der de La Mettrie'schen Idee des »'homme machine $\ll^{3}$. Leider haben zahlreiche Zeitgenossen auch dessen mechanistische Vereinfachung übernommen. Demgegenüber steht die Bescheidenheit der in diesem Gebiet aktiv Forschenden: Die Konstruktion des virtuellen Menschen erweist sich als extrem komplex und verlangt Interdisziplinarität von kaum gekanntem Ausmaß. Entlang dieser Entwicklungslinie können wir sicher mehr als die Teile, aber wohl nicht das geistige Band erwarten.

Physiker und Ingenieure entwickeln derzeit eine neue Generation von bildgebenden Verfahren. Sobald sie einsatzbereit sind, kann jedes Individuum über seine 3D-Daten verfügen, wenn es will und sich die Datenaufnahme leisten kann; die Preise werden natürlich bei Massenbedarf drastisch fallen. Ausreichend betuchte Leute haben schon heute ihre Schneidermaße an der Pariser Rive Gauche hinterlegt. In Zukunft werden sie ihre 3DDaten auf ihrer persönlichen Chipkarte mit sich führen und nach Bedarf bequem weitergeben können. Wir wer- 


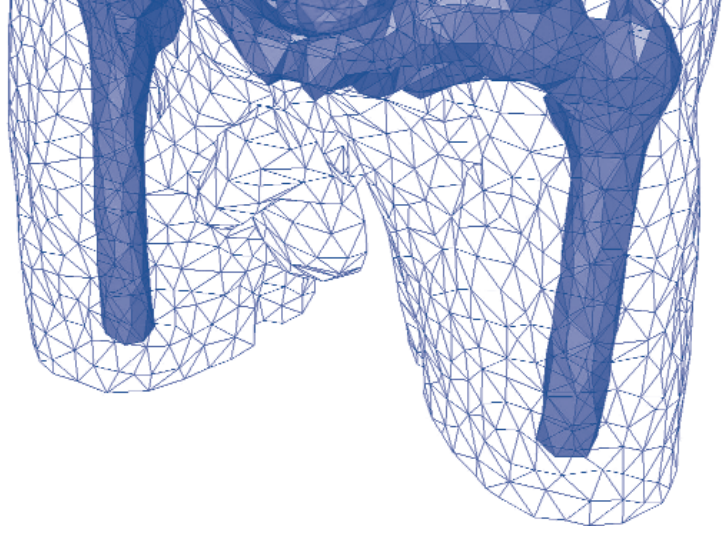

den auch noch den Couturier Virtuel konstruieren, vielleicht sogar für jede und jeden erschwinglich. Dies wäre nun ein schöner »Schluss für Leihbibliotheken« (Dürrenmatt). Dieselben mentalen Muster tauchen jedoch in ernstem Zusammenhang wieder auf: Die begriffliche Nähe zur Transplantationsmedizin springt ins Auge. Auch dort besteht die Gefahr, den Menschen nur als Summe seiner Teile oder bestenfalls als mechanistisches System zu imaginieren. Auch dort besteht Eile: Der Zelltod kann nicht abgewartet werden. Die individuelle postmortale Verfügungsgewalt erlischt mit dem Zeitpunkt des Hirntodes, definiert dadurch, dass ein Arzt »die Nulllinie schreibt «. In ihrem lesenswerten, gemeinsam mit Baureithel verfassten Buch zu dieser Thematik schreibt Bergmann: »Wie schon das Konzept des Hirntodes auf einem Körperbild basiert, in dem Organe aus ihrem Gesamtzusammenhang gerissen sind [...], so beruht auch die Therapie der Transplantation auf [...] demselben Prinzip der Fragmentierung. [...] Das >alte $<$ wie das >neue Organ werden dabei einem mechanistischen Prinzip der Austauschbarkeit unterworfen, das ein Organ ohne eigene Geschichte suggeriert.«(193, Kursivschreibung durch P. D.)

\section{In diesem Grenzgebiet unserer modernen Medizin} manifestiert sich das >geistige Band $\mathrm{des}$ menschlichen Körpers durch deutliche Signale.

* Die vehementen Abstoßungsbefehle des Immunsystems, das ja die individuelle Geschichte des Empfängers gespeichert hat, müssen für den verbleibenden Teil des Lebens durch Medikamente wie Cortison unterdrückt werden (Immunsuppression).

* Ein hoher Prozentsatz der Empfänger wie auch der Familienangehörigen der Spender hat mit massiven psychischen Belastungen zu tun, was zur Entstehung einer eigenen Transplantationspsychiatrie geführt hat. Das `Fremde im Eigenen` macht mentale Probleme, stößt sich am >magischen Vorstellungserbe des Menschen.

* Einzelne Wissenschaftler führen auf Basis unabweisbarer Beobachtungen, vollkommen auf sstofflicher Ebenes argumentierend, Begriffe wie Organgedächtnis oder Körpergedächtnis in die phänomenologische Beschreibung ein, gleichsam die Erinnerung von explantierten Organen an den gesamten Körper des Spenders. Auch jüngste Untersuchungen zur möglichen Existenz eines >Bauchhirns، - quasi eines verteilten Rechners als Pendant zum Zentralrechner Gehirn - passen in dieses Bild.
Ganz zuletzt wollen wir das Selbstverständliche nicht übersehen: Um ins Innere des menschlichen Körpers zu schauen, brauchen wir heute keine zum Tode Verurteilten mehr. Leichname von Hingerichteten haben im Prinzip ausgedient, sie können nun das gleiche Anrecht aufTotenruhe wie die von ehrbaren Bürgern beanspruchen. Mathematiker haben mitgeholfen, dass nunmehr auch aus dieser Sicht die Todesstrafe obsolet geworden ist. Allerdings haben zum Tode Verurteilte, im Zusammenhang mit Organspenden, doch wieder eine vertraute Rolle erhalten. In einer Dokumentation von 1994 hält die Organisation Human Rights Watch International der Volksrepublik China die erhöhte Zahl von Todesurteilen vor: Die Organe der Hingerichteten, unter denen sich zum großen Teil politische Dissidenten befinden, werden international verkauft (aber: nur an Chinesen).

Offenbar ist der Zusammenhang zwischen der Fragmentierung des Menschenbildes und der Zerstückelung von geometrischen 3D-Bildern doch enger als zunächst wahrgenommen.

\footnotetext{
Literatur

U. Baureithel und A. Bergmann: Herzloser Tod. Das Dilemma der Organspende. Stuttgart 1999

Chinese Visible Human Project: ttp: / /www.chinesevisiblehuman.com/ index-e.asp

P. Deuflhard: Therapieplanung an virtuellen Krebspatienten, in:

M. Aigner und E. Behrends (Hrsg.): Alles Mathematik. Braunschweig 2000, S. 22-30

Human Rights Watch / Asia. Vol. 6, No. 9. New York 1994, S. 2

P. J. Hunter und T. K. Borg: Integration from Proteins to Organs: the

Physiome Project, in: Nature Rev., Molecular Cell Biology 4, 2003,

S. 237-243

H. Marcuse: Der eindimensionale Mensch. München 1998

F. Natterer: The Mathematics of Computerized Tomography. New

York/Stuttgart 1986

Visible Korean Human: http://vkh3.kordic.re.kr/

M. Wadman: Ethics Worries over Execution Twist to Internet's

,Visible Man<, in: Nature 382, 1996, S. 657

F. Zöllner: Leonardo da Vinci. Sämtliche Gemälde und Zeichnungen. Köln 2003
}

1 VOXELMAN: http://www.uke.uni-hamburg.de/institute/imdm/idv/gallery/ index.en.html

2 Der nahe liegende Verdacht ist falsch: Es ist nicht der Autor.

3 Julien Offray de La Mettrie, auf Anweisung Friedrich II. seit 1748 Mitglied der

Preußischen Akademie der Wissenschaften 